\title{
Mathematical Explanation by Law
}

forthcoming in the British Journal for the Philosophy of Science

\section{Sam Baron}

\begin{abstract}
Call an explanation in which a non-mathematical fact is explained - in part or in whole - by mathematical facts: an extra-mathematical explanation. Such explanations have attracted a great deal of interest recently in arguments over mathematical realism. In this paper, a theory of extra-mathematical explanation is developed. The theory is modeled on a deductive-nomological theory of scientific explanation. A basic DN account of extra-mathematical explanation is proposed and then redeveloped in the light of two difficulties that the basic theory faces. The final view appeals to relevance logic and uses resources in information theory to understand the explanatory relationship between mathematical and physical facts.
\end{abstract}

1 Introduction

2 Anchoring

3 The Basic Deductive-Mathematical Account

4 The Genuineness Problem

5 Irrelevance

6 Relevance and Information

7 Objections and Replies

7.1 Against Relevance Logic

7.2 Too Epistemic

7.3 Informational Containment

8 Conclusion 


\section{Introduction}

Call an explanation in which a non-mathematical fact is explained by mathematical facts: an extra-mathematical explanation. ${ }^{1,2}$ The apparent prevalence of such explanations within science has attracted attention in the debate over the existence of mathematical objects. ${ }^{3}$ It is therefore surprising that few attempts have been made to develop a theory of scientific explanation that is capable of handling run-of-the-mill scientific explanations as well as cases of extra-mathematical explanation.

In this paper, I will not be concerned to argue for the existence of extra-mathematical explanations. There are plenty of such defenses available. ${ }^{4}$ Instead, I will assume that there are some such explanations and that a theory of the same is required. Importantly, I do not view it to be a goal of developing a theory of extra-mathematical explanation that such a theory should disabuse a sceptic about extra-mathematical explanations of her scepticism. No matter one's theoretical understanding of explanation there is always room for doubt, which is just as it should be.

A theory of extra-mathematical explanation will offer an account of the explanatory relation $-R-$ in virtue of which mathematical facts explain physical ones. The goal of this paper is to develop such a theory by extending the deductive-nomological theory of scientific explanation to handle extra-mathematical cases. ${ }^{5}$ On such a theory the $R$-relation is a relation of logical consequence. ${ }^{6}$ In Section Two I anchor the discussion by briefly considering some scientific uses of mathematics. In Section Three I present a basic DN account of extra-mathematical explanation. ${ }^{7}$ The theory is then refined in light of two problems (Sections Four and Five). In Section Six the refined DM theory is developed in greater detail and in Section Seven I consider some objections.

\section{Anchoring}

It is useful to begin by considering a clear case in which mathematics is explanatory and some clear cases in which it is not. We should, of course, be careful about putting too much stock in our intuitions about particular cases; intuitions should not be seen as hard boundaries for developing a theory. Nonetheless they can provide a basis for the start of enquiry by helping to delineate the target phenomenon. Delineating the target phenomenon with clear examples is important, since doing so is needed to tie the phenomenon to its analysis. Once a theory of the target phenomenon has been developed, then the relevant intuitions can be revisited, and

${ }^{1}$ This terminology is taken from Baker and Colyvan ([2011])

${ }^{2}$ Extra-mathematical explanations are typically explanations in which mathematics is playing an explanatory role in conjunction with physical facts.

${ }^{3}$ See Baker ([2005, 2009]); Baker and Colyvan ([2011]); Bangu ([2008]); Baron (2014, 2016a, 2016b); Baron and Colyvan ([2016]); Colyvan ([2001, 2002, 2010, 2012]); Lange ([2013]); Leng ([2010]); Lyon ([2012]); Lyon and Colyvan ([2008]); Mancosu ([2001]); Pincock ([forthcoming]); Saatsi ([2011, 2012, forthcoming]).

${ }^{4}$ See Baker ([2005], [2009], [2011]); Bangu ([2008]), Baron ([2014], [2016a], [2016b]); Baron and Colyvan ([2016]); Colyvan ([2001], [2002], [2010], [2012]); Lange ([2013]); Lyon ([2012]); Lyon and Colyvan ([2008]).

${ }^{5}$ Baker ([2005, 2009]) has suggested such an account; so has Lange ([2013]).

${ }^{6} \mathrm{DN}$-style theories of scientific explanation have been resuscitated by Jansson ([forthcoming]) and Strevens ([2008]).

${ }^{7}$ See Hempel $([1965,1948])$ for the original DN account. 
verdicts on cases where intuitions are unclear may be given.

The poster-child for extra-mathematical explanation is Baker's ([2005]) case of the North American magicicada. The case is by now well known and so I will be brief. Every thirteen or seventeen years - depending on the sub-species - the magicicada arises in its adult form for a period of around two weeks during which it eats, breeds, dies, and repeats the cycle. The phenomenon of magicicada swarming gives rise to an important explanatory question. Namely: why are the life-cycle lengths of the magicicada prime-numbered? The answer lies in number theory. Because of the way primes factorize, a prime-numbered life-cycle is the optimal strategy for avoiding predation. For instance, compare thirteen to twelve. Twelve has factors two, three, four, six, twelve, and one. So a cicada with a twelve-year life-cycle will overlap with predators that have periodical life-cycles of two, three, four, six, and twelve. Thirteen, by contrast, only has factors thirteen and one, and so will overlap only with predators that have thirteen-year life-cycles.

Further examples of extra-mathematical explanation are available. ${ }^{8}$ However, I will focus on the cicada case for three reasons. First, as an example of extra-mathematical explanation, that case has seen the most extensive defense to date. ${ }^{9}$ Second, the example has a good deal of intuitive pull. The mathematics really does seem to be playing a substantive role in the explanation. Third, the cicada case is an optimisation problem. Many of the examples of extra-mathematical available in the literature are problems of this kind (see Baron ([2014]) and Rice ([2015])). A theory of extra-mathematical explanation focused on the cicada case therefore promises to be widely applicable.

Compare the cicada case with the following example offered recently by Baron ([2016a], p. 451):

Suppose we want to explain why it is that a train $T$ arrives at a station, $S$, at 3:00pm. The explanation is as follows: $T$ left another station, $S *, 10$ kilometres away at 2:00pm and headed towards $S$ at $10 \mathrm{kph}$. Obviously, this explanation exploits some basic mathematics. Numbers are used to state the distance between stations as well as the speed of the train and a very basic mathematical calculation is deployed, namely: $\frac{10}{10}=1$.

Intuitively, the mathematics does not do any explanatory work in the explanation given. Rather, it is physical facts about the speed of the train and the distance between stations that fully explain why the train arrived when it did.

Steiner ([?]p. 142]steiner:am) outlines a similar case. Suppose we want to tile a rectangular floor. It takes 500 tiles to do so. Why 500 and not 501? The answer is simple: the floor is 50 units long and 10 units wide. The number of tiles needed is equivalent to $m \times n$ where $m$ is the

${ }^{8}$ Other examples of extra-mathematical explanation include explanations for: the shape of honeycomb cells (Lyon and Colyvan [2008]), the structure of seeds in a flower (Lyon [2012]), the search patterns of fully-aquatic marine predators (Baron [2014]), the minimal shape of Plateau's soap film (Lyon [2012]) the location of the Kirkwood gaps (Colyvan [2010]), the Fitzgerald-Lorenz contraction of bodies at relativistic speeds (Colyvan [2002]), the instability of high-energy galaxies (Lyon and Colyvan [2008]), the use of real-valued functions to understand physical systems (Peressini [1997]), and the use of Hilbert spaces in quantum mechanics as a basis for explaining quantum phenomena (Steiner [1995] and Peressini [1997]).

${ }^{9}$ See Baker ([forthcoming, 2009]); Baker and Colyvan ([2011]); Baron ([2014]) for four such defenses. 
length of the floor in units and $n$ is the width. Because $50 \times 10=500$ the number of tiles needed is 500 .

A third case has been offered by Melia:

[W] hen we come to explain [physical fact] $F$, our best theory may offer as an explanation ' $F$ occurs because $P$ is $\sqrt{2}$ metres long'. But we all recognize that, though the number $\sqrt{2}$ is cited in our explanation, it is the length of $P$ that is responsible for $F$, not the fact that the length is picked out be a real number. (Melia [2002], p. 76)

The mathematics in all three cases seems to be non-explanatory for the same basic reason: in each case the mathematics serves as a descriptive framework within which to express the explanation and within which to perform important calculations. ${ }^{10}$ As Peressini ([1997], pp. 218-219) argues, in cases such as these the mathematics is merely instrumental; it is not adding anything substantive to the explanation at hand.

\section{The Basic Deductive-Mathematical Account}

A theory of extra-mathematical explanation will provide an account of the explanatory relation that is present in cases like the cicada case and absent in cases like the train case. A DN approach to scientific explanation is a good place to look for such a theory. Many current theories of scientific explanation are tailored primarily toward modeling causal explanation. ${ }^{11}$ DN-style accounts, however, are not restricted to causal explanations. This is important as the relation in virtue of which mathematical facts explain physical ones is not causal. ${ }^{12}$ Moreover, as Colyvan (2010, 2012), Lyon ([2012]) and Lange ([2013]) have urged, extra-mathematical explanations typically show that the explanandum is necessary, for some modality or other. A theory of extra-mathematical explanation should be capable of handling the modal robustness of these explanations. The DN theory is well-placed to do so. By making relations of entailment carry explanations it is possible to imbue those explanations with the right kind of modal power. A DN theory of extra-mathematical explanation also offers unique prospects for unifying extra-mathematical explanation with intra-mathematical explanation (the explanation of one or more mathematical facts by others). One natural thought is that explanation within mathematics has something to do with proof on the one hand and the subsumption of particular mathematical facts under more general mathematical patterns on the other (see, for discussion, Lange, Lange ([2014, forthcoming])). If that's right, then a DN theory is a good bet. $^{13}$

According to the basic Deductive-Mathematical (DM) account, extra-mathematical explanation is a matter of subsuming explananda under mathematical facts. As in the standard DN account, the subsumption of an explanandum under mathematical facts is modeled via the deduction of non-mathematical claims in part from mathematical claims. The basic DM

\footnotetext{
${ }^{10}$ For further discussion of the role of mathematics in describing or 'indexing' physical phenomena see Baker and Colyvan ([2011]); Saatsi ([2011, forthcoming]).

${ }^{11}$ See Salmon's ([1984]) process theory and the counterfactual theory defended by Woodward (2003), Woodward and Hitchock (2003), Lewis (1986) and Skow ([forthcoming]). Some philosophers suggest ways of extending their accounts to handle non-causal explanations in science. See e.g., Woodward ([2003]) and Salmon ([1984]).

${ }^{12}$ Lyon (2012) makes this point.

${ }^{13}$ For discussion of intra-mathematical explanation see Colyvan et al. ([2017]).
} 
account can be stated as follows: ${ }^{14}$

The Basic DM Theory of Extra-Mathematical Explanation

1 Extra-mathematical explanations are sound arguments.

2 The conclusion of an extra-mathematical explanation is a proposition stating the physical phenomenon to be explained.

3 Among the premises of an extra-mathematical explanation there must be at least one mathematical claim.

4 If the mathematical claim were removed from the premises of an explanatory argument, then the argument would become invalid.

It is useful to apply the theory to an actual case. It is straightforward to formulate Baker's cicada case as a deductive argument (see Baker ([2005], p. 233)):

[P1] Having a life-cycle period which minimises intersection with other (nearby / lower) periods is evolutionarily advantageous.

[P2] Prime periods minimise intersection (compared to non-prime periods).

[P3] If having a life-cycle period which minimises intersection with other (nearby / lower) periods is evolutionarily advantageous, and prime periods minimise intersection (compared to non-prime periods) then organisms with periodic life-cycles are likely to evolve periods that are prime.

[P4] Cicadas in ecosystem-type, $E$, are limited by biological constraints to periods from fourteen to eighteen years.

[P5] If organisms with periodic life-cycles are likely to evolve periods that are prime and cicadas in ecosystem-type, $E$, are limited by biological constraints to periods from fourteen to eighteen years, then cicadas in ecosystem-type, $E$, are likely to evolve seventeen-year periods.

[P6] Cicadas in ecosystem-type, $E$, are likely to evolve seventeen-year periods.

The argument satisfies the tenets of the basic DM theory and as such is classified as a genuine case of extra-mathematical explanation, which is the right result. In what follows I will refine the basic DM account by addressing a series of difficulties for the view. The result will be a more robust theory that may be used as a basis for future work.

\footnotetext{
${ }^{14}$ A note on terminology: a 'mathematical claim' is a true or false proposition about a mathematical fact, such as the claim that there are an infinite number of primes. Mathematical claims are represented by sentences that use mathematical language. The deduction of a non-mathematical claim from mathematical claims will involve a group of premises that are constituted by mathematical sentences expressing those claims and a conclusion that is constituted by a non-mathematical sentence (a sentence using no mathematical language) that expresses a non-mathematical claim (such as a proposition about empirical reality).
} 


\section{The Genuineness Problem}

Here's the first problem. It is a straightforward matter to formulate cases like the train case outlined in Section Two as deductive arguments that satisfy the core tenets of the basic DM account:

[P1] $T$ left $S *$ at 2:00 pm.

[P2] $S *$ is 10 kilometres away from $S$.

[P3] $T$ is travelling at $10 \mathrm{kph}$.

[P4] For any number $\mathrm{m} \frac{m}{m}=1$.

[P5] If for any number $\frac{m}{m}=1$, then $\frac{10}{10}=1$.

[P6] If $T$ left $S *$ at 2:00 pm, $S *$ is 10 kilometres away from $S, T$ is travelling at $10 \mathrm{kph}$, and $\frac{10}{10}=1$, then $T$ arrives at $S$ at 3:00pm.

[P7] $T$ arrives at $S$ at 3:00pm.

By formulating the train case in this fashion the point is not that the DM theory incorrectly classifies the case as an extra-mathematical explanation, thereby violating our intuitions (though it certainly does that). The point, rather, is that the ease with which such an argument may be formulated is telling. A little reflection reveals that most (if not all) uses of mathematics in science can be formulated into an argument in much the same manner. So the basic DM theory lacks the resources to uphold a meaningful distinction between explanatory and non-explanatory uses of mathematics in science. Call this: the genuineness problem.

In order to solve the genuineness problem, a further constraint on the basic DM account is needed. Here's my suggestion: claims concerning the non-mathematical facts that one is attempting to explain must be essentially deducible from the mathematical claims at issue. This 'essential deducibility' constraint may be formulated as follows (note that a 'physical sentence' is a sentence that expresses a claim about physical reality and that uses no mathematical language):

Essential Deducibility Constraint [EDC]: A non-mathematical claim $P$ is essentially deducible from a premise set $S$ that includes at least one mathematical sentence $M$ just when there is a sound derivation of $P$ from $S$ and there is no sound derivation of $P$ from a premise set $S *$ that includes only physical sentences.

When a non-mathematical claim is essentially deducible from a set of premises that includes mathematical claims, the argument is a genuine extra-mathematical explanation. When the non-mathematical claim is not essentially deducible in this manner, because there is an alternative derivation that includes only physical claims, the derivation of the non-mathematical claim in part from mathematical claims is not a genuine extra-mathematical explanation.

Some fine-tuning of the constraint is called for. What it is for mathematics to be playing a genuinely explanatory role in science can't simply be a matter of there being no alternative way of getting to the physical facts. Rather, presumably even if an alternative route is available, it may sometimes be the case that the way via mathematics is superior to the non-mathematical way. This idea may be captured by sharpening the essential deducibility constraint as follows:

Sharp Essential Deducibility Constraint [SEDC]: A non-mathematical claim $P$ is essentially deducible from a premise set $S$ that includes at least one mathematical 
sentence $M$ just when there is a sound derivation of $P$ from $S$ and either there is no sound derivation of $P$ from a premise set $S *$ that includes only physical sentences or all sound derivations of $P$ from premise sets $S_{1} \ldots S_{n}$ each of which includes only physical sentences are worse than the mathematical derivation.

The SEDC presumes that derivations can be ranked in terms of overall quality. For the constraint to yield the desired result derivations involving mathematics must dominate at least some such orderings. To give the SEDC content something must be said about what the relevant ranking is. We need criteria by which derivations may be ranked; criteria that reflect the good-making features of the physical or scientific explanations that the derivations encode. Exactly what these criteria are is therefore beholden to our best account of the good-making features of physical or scientific explanations. What those good-making features are is controversial. For now it will be sufficient to show how one might rank derivations with respect to two of the most widely accepted good-making features of such explanations: simplicity and unity.

Here's the idea. A sound argument $A$ for conclusion $C$ is simpler than a sound argument $A *$ toward the same conclusion when $A$ has fewer distinct premises than $A *$. A sound argument $A$ for conclusion $C$ has a greater degree of unity than a sound argument $A *$ toward the same conclusion when $A$ 's premises may be used to establish a greater range of conclusions other than $C$ than can $A$ *'s premises. The best derivations are those that strike the optimal balance between simplicity and unity understood in this sense. The arguments that strike this balance represent those explanations that are the simplest and that have the most unificatory power, which will be among the best explanations for the phenomenon of interest. Note that the move here is by no means new: the trade-off is similar to the one found in Lewis's ([1994]) best-systems account of law-hood. Unity as I use it here is Lewis's notion of strength.

Both simplicity and strength (unity) are contested notions. So I need to say a bit more. First: simplicity. Simplicity has been the subject of a great deal of criticism in the philosophy of science. In the wake of this critique, an important strategy for justifying simplicity has emerged. This strategy is broadly probabilistic in nature: simpler theories are more likely to be true.

One version of the probabilistic strategy - the likelihood account - may be sketched as follows. First, given Bayes' theorem, the probability of a theory $T$ (simple or complex) given evidence $E$ is:

$$
P(T \mid E)=\frac{P(T) \times P(E \mid T)}{P(E)}
$$

Next, note that simpler theories are compatible with fewer items of evidence. This is because simpler theories tend to have fewer adjustable parameters. With fewer adjustable parameters, there is less scope for a theory to be modified so that it fits the data (see Huemer ([2009], pp. 221-222) and Forster and Sober ([1994], pp. 10-11). Suppose, then, that we have a simple theory $S$ and a complex theory $C$. Suppose, further that $S$ is compatible with and neutral between two items of evidence $E_{1}$ and $E_{2}$, whereas $C$ is compatible with and neutral between four items of evidence $E_{1}, E_{2}, E_{3}$, and $E_{4}$. Now, consider a particular piece of evidence, $E_{1}$. Suppose that $E_{1}$ and only $E_{1}$ is observed. Which theory is more likely to be true? Well, $P\left(E_{1} \mid S\right)=\frac{1}{2}$, whereas $P\left(E_{1} \mid C\right)=\frac{1}{4}$. Assuming the prior probabilities of $S$ and $C$ are equal, it follows by $[\mathrm{A}]$ that $P(S)>P(C){ }^{15}$

\footnotetext{
${ }^{15}$ This example is taken from Huemer ([2009], pp. 221-222).
} 
This is not the only probabilistic justification for why it is that simpler theories tend to be more likely. A range of other strategies have been offered and developed in detail by Sober ([2016]). ${ }^{16}$ I won't survey these strategies here. All that matters is that the SEDC uses a type of simplicity that is ultimately open to probabilistic justification, since then it is in line with the use of simplicity in science more generally. And indeed it does: the type of simplicity used by the SEDC concerns the number of distinct premises within a deduction. Translated into the explanatory key of the DM theory, the number of premises corresponds to the number of distinct claims that constitute an explanation. Essentially, an explanation is simpler when it makes fewer claims. An explanation that makes fewer claims, however, will be compatible with fewer items of evidence. By making fewer claims, it has fewer adjustable parameters and so will be harder to fit to the data. By the same Bayesian reasoning, then, explanations that are simpler in the sense just described are more likely to be true. So the likelihood account applies. But so too will other probabilistic justifications, since those justifications typically apply to the type of simplicity under discussion.

This brings us to strength. There is a well-known problem with Lewis's notion of strength. The problem is that the strength of a deduction is beholden to the system of basic predicates that is used to underwrite the deduction Cohen and Callender ([2009], p. 6). If the strength of a deduction is beholden to a choice of expressive resources, then it will be difficult to compare DM arguments in terms of their strength without already settling on a choice of basic predicates. But how do we do that? Lewis ([1983]) maintained that nature provides us with a privileged class of predicates. The predicates in question are those that correspond to the perfectly natural properties.

There are two problems with Lewis's solution. First, the perfectly natural properties are, for Lewis at least, the fundamental properties. This makes it very difficult to understand strength in the context of sciences that deal in the non-fundamental (Schrenk [2014], pp. 1791-1792). Second, it is unclear how to extend the concept of a perfectly natural property to mathematics. One option is to appeal to fundamental properties again. But extra-mathematical explanations often deal with non-fundamental mathematical properties (such as certain number theoretic properties).

Cohen and Callender ([2009]) offer an alternative to Lewis's solution. Let strength be relativized to a choice of basic predicates. Let the choice of basic predicates be set by the science within which the explanation is formulated. So, for example, within biology, let the basic predicates be biological ones; within physics, let the basic predicates be the predicates of fundamental physics and so on. Having relativized strength in this manner, conduct the trade-off between strength and simplicity with respect to that choice. Finally, accept that there are many strongest systems of derivation, each relativized to a scientific domain.

Cohen and Callender's solution can be extended for use by the DM theory. Let us allow that basic mathematical predicates form part of the expressive stock for determining strength. With respect to derivations involving mathematics, we then let the choice of basic predicates be partially set by the area of mathematics being used and partially set by the domain of science. So, for instance, if one is working in biology using number theory, then let the basic predicates be those found in biology and number theory; if one is working in physics and graph theory, then let the basic predicates be physical and graph-theoretic and so on. The strength of derivations can then be compared with respect to a choice of basic predicates, with the strongest being the best for a mixed scientific/mathematical domain.

How much of a mathematical theory is required in order to delimit the mathematical

\footnotetext{
${ }^{16}$ See also Jansson and Tallant ([2016]).
} 
vocabulary at issue? It may be that the full pure mathematical theory is invoked. For example, Steiner ([1995], p. 149) and Peressini ([1997], p. 221) both argue that for some mathematical explanations of quantum phenomena, the entire Hilbert space formalism that underwrites the explanation will be needed. ${ }^{17}$ If that's right and, moreover, the full pure mathematical theory is generally required, then the deductions at issue may invoke all of the pure mathematical inferences that correspond to the relevant pure theory. A case in which a physical claim is deduced from mathematics may therefore be very strong indeed. But this kind of strength is not of the right type for comparing mathematical explanations of physical phenomena. We want to compare these explanations in terms of the consequences they have for physical claims only. Accordingly, a further modification to the Cohen and Callender solution is required. The notion of strength at issue must be restricted to the deduction of non-mathematical claims. This prevents the purely mathematical implications from introducing unwanted noise into our evaluation of the relative strength of DM arguments.

While adopting Cohen and Callender's view helps, doing so gives rise to another difficulty. Suppose we want to explain a particular phenomenon $P$. There are two choices of basic predicates available for doing so. One choice uses purely physical language. The other uses a mixture of mathematical and physical predicates. Now, suppose that a derivation of $P$ is possible with respect to both choices. In the purely physical case, the strongest derivation of $P$ uses only physical predicates; in the mixed mathematico-physical case, the strongest derivation uses mathematical and physical predicates. In this situation, the mathematics might not be playing an explanatory role; for there is an explanation available for $P$ that makes no use of mathematics. However, because the two derivations are conducted using different expressive resources, the strength of the two deductions cannot be compared. Because the strength of the two derivations cannot be compared, we cannot say that one explanation is better than the other, and so we cannot determine whether the mathematical derivation is better than the non-mathematical one. So we don't know if the mathematics is playing a genuine explanatory role. The SEDC doesn't apply in this case. But this is exactly where it should apply.

To address this problem I propose a further modification to the SEDC:
Razor-Sharp Essential Deducibility Constraint [REDC]: A non-mathematical claim $P$ is essentially deducible from a premise set $S$ that includes at least one mathematical sentence $M$ just when for an appropriate choice of expressive resources there is a sound derivation of $P$ from $S$ and either for the same choice of expressive resources there is no sound derivation of $P$ from a premise set $S *$ that includes only physical sentence or all sound derivations of $P$ from premise sets $S_{1} \ldots S_{n}$ each of which includes only physical sentences are worse than the mathematical derivation or for all appropriate choices of expressive resources the best derivations use $M$.

We supervaluate over appropriate choices of expressive resources; a choice is 'appropriate' when it is licensed by the mathematical or scientific domains within which the explanation is formulated. When $M$ dominates no matter which choice of basic predicates is used, then $M$ is genuinely explanatory. If, however, for a choice of expressive resources, there is a way of deriving $P$ that makes no use of $M$ and that bests any derivation that uses $M$, then $M$ is not explanatory.

${ }^{17}$ The more general issue of how much of a pure theory is needed in a given case of explanation is discussed in Peressini ([2010]) and Steiner ([1992]). 
While the REDC provides a more nuanced account of strength and simplicity, it faces a further difficulty. Consider a DM argument in which $P$ is derived, in part, from a mathematical claim $M$. Call the argument $\alpha$. Suppose $\alpha$ satisfies the REDC. Suppose, also, that this is sufficient for $\alpha$ to count as a genuine extra-mathematical explanation. In virtue of satisfying the REDC, it also follows either that there is no alternative derivation (and thus explanation of) $P$ that does not feature $M$ or that all alternative derivations that do not feature $M$ are inferior (either for a choice of expressive resoures, or across all choices). But $M$ is indispensable to an explanation when these same conditions are met. So, by REDC, any case of indispensability for $M$ is also a case of genuine extra-mathematical explanation. This leaves no room for $M$ to be indispensable to an explanation, and yet for $M$ to play a merely descriptive role in that explanation. In short, the REDC threatens to collapse the distinction between descriptive and explanatory indispensability (and thus between explanation and indispensability more generally).

To avoid this outcome, a further constraint must be imposed on the basic DM theory. In order to state the constraint, however, I require the notion of informational containment developed in Sections Four and Five, which is used to address the second problem facing the basic DM theory. Let us therefore set aside the genuineness problem for a moment and return to it with a concept of informational containment in hand.

\section{Irrelevance}

The second problem facing the basic DM theory focuses on relevance. Consider the following argument:

$\left[\mathrm{P} 1^{*}\right]$ Having a life-cycle period which minimises intersection with other (nearby / lower) periods is evolutionarily advantageous.

$[\mathrm{P} 2 *] 2+2=4$.

[P3*] If having a life-cycle period which minimises intersection with other (nearby / lower) periods is evolutionarily advantageous, and $2+2=4$ then organisms with periodic life-cycles are likely to evolve periods that are prime.

[P4*] Cicadas in ecosystem-type, $E$, are limited by biological constraints to periods from fourteen to eighteen years.

[P5*] If organisms with periodic life-cycles are likely to evolve periods that are prime and cicadas in ecosystem-type, $E$, are limited by biological constraints to periods from fourteen to eighteen years, then cicadas in ecosystem-type, $E$, are likely to evolve seventeen-year periods.

[P6*] Cicadas in ecosystem-type, $E$, are likely to evolve seventeen-year periods.

All I have done is swap one mathematical claim for another. Since the mathematical claim expressed in $[\mathrm{P} 2 *]$ is true, $[\mathrm{P} 2 *]$ and $[\mathrm{P} 3 *]$ are both true, and since the logical form of the argument remains intact, the argument is valid (and so sound if the original argument is). The argument also satisfies the core tenets of the basic DM account, since the explanandum is derived, in part, from mathematics. Finally, the argument satisfies the REDC just when Baker's original argument does. So it seems we are forced to treat the above argument as one that represents a genuine extra-mathematical explanation. And yet that is the wrong result: the mathematical claim at $[\mathrm{P} 2 *]$ - that $2+2=4-$ is irrelevant to cicada life-cycle length.

Call this the irrelevance problem. Note that, as with the genuineness problem, the worry is 
not that the DM theory contradicts our intuitions about what is relevant and what is not for a given explanation (though it certainly does that). The problem is that any and all mathematical claims can be added to the cicada case (or, indeed, any DM argument) in the same manner. But it is not the case that all mathematical claims are relevant to every explanandum. So something has gone wrong: the DM theory lacks the capacity to sort relevant mathematical claims from irrelevant ones when it comes to explanation.

The difficulty is quite similar to a familiar problem facing the standard DN account of scientific explanation. For instance, consider the following argument outlined by Salmon (1971, p. 34):

[P1] All males who take birth control pills regularly fail to get pregnant.

[P2] John Jones is a male who has been taking birth control pills regularly.

[P3] John Jones fails to get pregnant.

Clearly, the above argument does not represent a genuine scientific explanation. John Jones's failure to get pregnant is not explained by his birth-control regimen. Standard DN accounts must be modified in order to handle this kind of case. The problem facing the basic DM account is that the standard strategies available for addressing the John Jones case are not available for ruling out arguments like the specious cicada argument and so cannot be used to differentiate relevant from irrelevant mathematical claims.

There are, broadly speaking, two such strategies. The first is to apply a further, causal condition of adequacy over DN arguments. So, for instance, in the John Jones case, it is not true that taking birth control pills causes John Jones's failure to get pregnant. It is, rather, the fact that he lacks the physiological traits needed to get pregnant that causes his failure to get pregnant. Accordingly, it is possible to rule that the John Jones argument is not a genuine scientific explanation because only those arguments that correspond to the causal facts of the explanatory situation are genuine scientific explanations.

The second strategy is to provide a more precise account of what it is to be a law of nature. The John Jones case relies on one's account of what it is to be a law of nature being sufficiently broad to include the generalisation that all males who take birth control pills regularly fail to get pregnant. By supplementing the standard DN account with a more stringent account of what it is to be a law, one may be able to rule that the first premise of the John Jones case does not, in fact, correspond to a law. This would then disqualify the argument from being an explanation.

Evidently, the causal strategy is of no use in patching up the basic DM theory. There is no difference in the causal facts being tracked by Baker's cicada argument as compared to an argument like the specious cicada argument outlined above. In so far as either argument tracks the causal facts of the situation, they both do. There is no further causal relationship between, say, prime numbers and cicada life-cycle length that Baker's cicada argument captures but that the specious argument misses. Indeed, a moment's reflection reveals that no causal constraint whatsoever can be used to solve the irrelevance problem for the basic DM theory: mathematical facts do not cause physical facts and so there is no causal difference between two DM arguments that differ only with respect to the mathematical claims they deploy.

Providing a more precise account of what it is to be a law of nature won't obviously help either, since the difficulty posed by arguments like the specious cicada argument has nothing to do with the laws. It is not because ' $2+2=4$ ' fails to be a law, whereas 'prime periods minimise intersection (compared to non-prime periods)' is a law that we can rule against 
arguments like the specious cicada argument. Neither of these claims are laws of nature in the relevant sense, they both correspond to mathematical facts. So there is no way to sort relevant from irrelevant mathematics by tightening the notion of a law.

Now, one might seek to broaden the concept of a physical law of nature to include statements that feature mathematical claims. Indeed, Baker ([2005]) makes a suggestion along these lines. So, for instance, compare [P3] with [P3*] (repeated below):

[P3] If having a life-cycle period which minimises intersection with other (nearby / lower) periods is evolutionarily advantageous, and prime periods minimise intersection (compared to non-prime periods) then organisms with periodic life-cycles are likely to evolve periods that are prime.

[P3*] If having a life-cycle period which minimises intersection with other (nearby / lower) periods is evolutionarily advantageous, and $2+2=4$ then organisms with periodic life-cycles are likely to evolve periods that are prime.

It could be argued that while [P3] is a candidate to be a physical law of nature, [P3*] is not. In order to turn this move into a solution to the problem at hand, however, one must strengthen the DM theory with the following requirement:

( $\left.3^{*}\right)$ Among the premises of an explanation there must be at least one mathematical claim and it must feature in a law of nature.

By strengthening the DM theory with $\left(3^{*}\right)$, it may be possible to rule out arguments like the specious cicada argument: that argument is not an extra-mathematical explanation because [P3*] is not a law of nature, and ' $2+2=4$ ' does not feature in any other laws of nature within that argument. There are, however, two difficulties with this suggestion. First, as Baron ([2016a], pp. 466-467) has argued, the same problem can be formulated for the laws themselves. We can always take a putative law like [P3] and replace the mathematics in [P3] with something irrelevant to produce a claim that is just as good a candidate to be a law of nature. We just do not have an account of physical laws available that will rule out [P3*] while ruling [P3] in. Second, $\left(3^{*}\right)$ may be too strong a requirement to place on the DM theory. As Lange ([?]p. 505]lange:wmsedm) has argued, one of the distinctive features of extra-mathematical explanations is that they have the capacity to explain physical phenomena without involving any laws of nature. Such explanations can therefore show an explanandum to hold even if the laws were different. If the mathematics in an extra-mathematical explanation must always be routed through the laws, however, then this distinctive feature of extra-mathematical explanation will be lost. At best, such an explanation can show an explanandum to hold of physical necessity.

It seems, then, that standard solutions to irrelevance worries for traditional DN accounts of explanation won't generalize to the DM account. So a new solution is required. Here's my (somewhat radical) proposal: to solve the problem, modify the logical foundations of the DM theory. Instead of formulating the DM theory in the same logical terms as the DN theory namely, using classical logic - we may instead formulate the DM theory using a relevance logic. In particular, the relevance logic $\mathbf{R} .{ }^{18,19}$ Relevance logic places a relevance constraint on

\footnotetext{
${ }^{18} \mathbf{R}$ is needed because $\mathbf{R}$ and its fellows make use of the containment relation 'ㄷ', which is needed to handle the notion of informational containment described in Section Six.

${ }^{19}$ The use of relevance logic as a basis for understanding mathematical explanation has already been suggested by Colyvan et al. ([2017]) in the context of intra-mathematical explanation.
} 
both the semantic relation of consequence that connects premises to conclusions and the conditional ' $\rightarrow$ '. Within relevance logic, $\Gamma \models \Delta$ only if $\Gamma$ is relevant to $\Delta$ and $\Gamma \rightarrow \Delta$ only if $\Gamma$ is relevant to $\Delta .{ }^{20}$ Exactly what it means to say that $\Gamma$ is relevant to $\Delta$ will be considered in greater detail in the next section. For now, it is enough to see how shifting toward a relevance logic helps with the irrelevance problem.

Consider, again, the specious cicada argument outlined above. This argument seems specious precisely because ' $2+2=4$ ' does not appear to be relevant to the life-cycle length of the periodical cicadas. A relevance logic has the potential to satisfy this basic idea. As we have seen, if [P3] in Baker's formulation of the cicada argument is swapped for [P3*], and $[\mathrm{P} 2]$ for $[\mathrm{P} 2 *]$ a sound argument is produced (so long as the argument with [P3] and [P2] was already sound). But while classical logic is blind to the difference between [P3] and [P3*]; a relevance logic is not. A relevance logic vindicates [P3] but not [P3*]: [P3] is true because each part of the antecedent is relevant to the consequent; [P3*] is false because some part of the antecedent is irrelevant to the consequent. Note that to deliver this result, what we must demand is that the antecedent be wholly relevant to the conclusion, in this sense: no part of the antecedent is irrelevant to the consequent. The same demand may be imposed on the consequence relation from premise to conclusion, as follows: no part of the set of premises is irrelevant to the conclusion. Consequence under this demand is no-longer simply a matter of necessary truth-preservation from premise to conclusion. Rather, consequence is now a matter of relevantly constrained necessary truth-preservation: both truth and relevance must be preserved from premise to conclusion.

Call an argument that obeys the strictures of a relevance logic, an $\mathbb{R}$-Argument, then according to the refined DM theory of extra-mathematical explanation, extra-mathematical explanations are sound $\mathbb{R}$-Arguments. The refined DM theory of extra-mathematical explanation has an added advantage. Explanations are non-monotonic. If $A$ explains $B$, then it is not the case that $A$ and $C$ explain $B$, for any $C$ whatsoever. Classical consequence, however, is monotonic. So one can produce all kinds of junk arguments that satisfy the strictures of the basic DM account simply by exploiting the monotonicity of the classical consequence relation. The consequence relation of relevance logic is non-monotonic. So the logical foundations of the refined DM theory accord more closely with the logical features of explanation more generally. It is a straightforward matter to demonstrate the non-monotonicity of relevance logic, but I will spare the reader the technical details. ${ }^{21}$ Instead, we can understand in an informal sense why a relevance logic is non-monotonic as follows. Suppose, as I have suggested, that the premises need to be wholly relevant to the conclusion: no part of the premise set is irrelevant to the conclusion. Well, it follows that one cannot add premises to the premise set willy-nilly and expect validity to be preserved. For if one adds an irrelevant premise, then the validity of the argument will be undermined, since the premises will no-longer be wholly relevant to the conclusion. So the logic is non-monotonic. ${ }^{22}$

${ }^{20}$ See Andersen and Belnap ([1975]); Goldblatt and Mares ([2006]); Mares ([2009]); Mares ([2004]); Priest ([2002]) and Restall ([1996]) for the formal details.

${ }^{21}$ Relevance logics are non-monotonic because they jettison weakening. See Restall and Dunn ([2002]).

${ }^{22}$ Using relevance logic because it is non-monotonic mirrors a solution offered by Waters ([1987]) to the irrelevant conjunction problem for hypothetico-deductivism, which may be stated as follows (see Skyrms ([1992]) for an overview of the problem). For background theories $T$, for hypothesis $H$ and evidence $e$ if $e$ confirms $H$ then: (i) $T$ and $H$ are consistent, (ii) $T, H \vdash e$, and (iii) $T \nvdash e$. When $T, H$ and $e$ satisfy these three conditions, for any $A$ whatsoever, $T,(H \& A)$ and $e$ also satisfy the conditions. Waters proposes to address the 
As it stands, the refined DM theory of extra-mathematical explanation may not seem particularly plausible. Consider the concept of relevance that is at work in the statement of the refined DM theory. What is it for the antecedent of a conditional to be relevant to its consequent? Similarly, what is it for the premises of an argument to be relevant to their conclusion? Here's one answer: $\Gamma \models \Delta$ only if $\Gamma$ is explanatorily relevant to $\Delta$ and $\Gamma \rightarrow \Delta$ only if $\Gamma$ is explanatorily relevant to $\Delta$. If this answer is correct, then the refined DM theory of extra-mathematical explanation is no good. Recall that our goal in producing a theory of extra-mathematical explanation was to provide some account of the relation, $R$, in virtue of which mathematical facts explain non-mathematical facts. But if the notion of relevance that is operative in a relevance logic just is explanatory relevance, then it would seem that an account of $R$ is the very thing we need to interpret the concept of relevance that the logic deploys. In short, if 'relevance' is understood to be 'explanatory relevance' then the refined DM theory is painfully circular.

In the following section I will outline one, recent interpretation of the relevance relation that does not treat relevance as explanatory relevance. In Section Seven I will then raise and address two objections for that interpretation when it is used as a basis for clarifying the refined DM theory of extra-mathematical explanation. First, however, it is worth pausing to consider one option for understanding relevance that I won't pursue. This is to understand relevance syntactically. A syntactic understanding of relevance treats relevance entirely in terms of variable sharing. Very roughly, on such a view, what it is for the premises of an argument to be relevant to the conclusion of that argument is for there to be (at least one) propositional variable that appears in both the premises of the argument and the conclusion of that argument. A variable sharing understanding of relevance for DM arguments might therefore be provided as follows: it is a necessary condition over DM arguments that at least some of the mathematics appearing in the premises of those arguments also appears in the conclusion. This way of interpreting relevance will successfully rule out arguments like the spurious cicada argument outlined above, but it ultimately won't do. Many cases of extra-mathematical explanation don't feature any mathematics in the conclusion. ${ }^{23}$ So variable sharing of the kind described for mathematical claims may not be assured. Indeed, as Bangu ([2008]) has argued, if mathematical facts do appear in the explanandum of a putative extra-mathematical explanation, then that explanation is left open to the charge that it is actually an intra-mathematical explanation: an explanation of one mathematical fact by another. ${ }^{24}$ It follows that in order for the DM theory to correctly handle extra-mathematical explanations as such, an alternative account of relevance is needed.

\section{Relevance and Information}

In providing an interpretation of the concept of relevance that appears in relevance logic the aim is not to provide a semantics for relevance logic. The standard semantics for relevance

irrelevant conjunction problem by using relevance logic, rather than classical logic, as the basis for hypothetico-deductivism.

${ }^{23}$ Colyvan's (2010) example of the Kirkwood gaps is like this.

${ }^{24}$ Baker ([2009]) recognizes this difficulty and, in recent work, has reformulated the cicada case without any mathematical facts in the explanandum. 
implication is the Routley-Meyer ternary semantics. ${ }^{25,26}$ Rather, by 'interpretation' I mean a philosophical account of what it means to say that the premises of an argument must be relevant to its conclusion, and the antecedent of a conditional must be relevant to its consequent. There is no one interpretation of relevance that all logicians accept. Nevertheless, it is possible to discern a common thread that runs through extant interpretations; an element that constitutes something of a consensus about how to understand relevance implication. This is the idea that relevance is to be understood in terms of information. The kind of information at issue is semantic information - information about something — and has its roots in the information theory coming out of the work of Dretkse ([1981]), Israel and Perry ([1990]), and Barwise and Seligman ([1997]).

There are various ways to interpret relevance logic through the lens of information theory. It is not necessary to lay out the full details of each informational account of relevance logic here. For now, I will proceed with a rough and rather broad account of relevance logic in terms of informational containment. What it means to say both that $\Gamma \models \Delta$ only if $\Gamma$ is relevant to $\Delta$ and that $\Gamma \rightarrow \Delta$ only if $\Gamma$ is relevant to $\Delta$ is this: (i) all of the information contained within $\Delta$ is contained within $\Gamma$ and (ii) each member of $\Gamma$ contains some part of the information in $\Delta$. A proposition gets its informational content from the part of the world that makes it true. So, for instance, the proposition that the window is smashed by the rock contains information about window smashings and rocks, and gains that information by being made true by a particular window-smashing event. Similarly, mathematical claims such as $2+2=4$ get their information from the mathematical facts about two and four that make that claim true.

What is semantic information metaphysically speaking? There isn't much consensus on this either. However, despite lacking a fully worked-out metaphysics for semantic information, the notion receives rather heavy use in relevance logic and information theory nonetheless. I hope I won't be too disparaged as I join the multitudes. Besides, semantic information is clearly something that we all have some grasp on. Semantic information is what we're talking about when we say things like: 'my map provides information about the whereabouts of local bars' or 'this novel contains information about the history of World War II' or 'your description of the solar system gives me a lot of astronomical information to work with' or even 'what you just said was very informative'. It's that kind of information that is being used to interpret the concept of relevance, and it is that kind of information that is ultimately underwriting the refined DM theory of extra-mathematical explanation. Note also that the concept of explanatory information already has currency in the literature on scientific explanation. ${ }^{27}$ Explanatory information is just a particular kind of information, namely the kind of information that features in explanations. What the refined DM theory does is provide a partial account of what kind of information explanatory information is: it is information that features in relationships of informational containment where those relationships involve

${ }^{25}$ See Routley and Meyer ([1973]); Routley and Meyer ([1972a]) and Routley and Meyer ([1972b]). For discussion see Beall et al. ([2012]).

${ }^{26}$ The truth-conditions for the relevant ' $\rightarrow$ ' are to be analysed in terms of a ternary accessibility relation $R x y z$ over worlds $u, v, w$ (the semantics extends in the usual way to $\Gamma \vDash \Delta)$ :

$w \vDash \phi \rightarrow \psi$ if and only iff for each $u, v$ where $R u w v$, if $u \vDash \phi$ then $v \vDash \psi$. (Restall 1996a, p. 464)

${ }^{27}$ E.g., the 'explanatory store' in Kitcher ([1989]) and the 'ideal explanatory text' in Railton $([1978,1981])$. 
mathematical facts essentially.

What is informational containment? As before, no agreed-upon theory is available but, again, a basic grip on this notion can be easily gained. Informational containment is the phenomenon we have in mind when we say things like 'my map and your map contain the same information' or 'the information provided by the defendent does not contain the same information as that provided by the prosecution' or 'the second edition contains all of the information about World War II that the first edition contained, plus some extra information about Russia's involvement'. Informational containment is a relation between pieces of information, which we may call: information states. An information state $i$ contains the information in a distinct information state $j$ when information state $j$ is a part of information state $i$.

There is a great deal more to say about semantic information and about informational containment, but I won't attempt to say it here. All that matters is that both semantic information and the relation of informational containment are phenomena that are fairly familiar to us and to which we often appeal. So despite lacking a theory of these phenomena, I see no problem with making use of informational containment in the present context. Indeed, issues to do with the nature of semantic information and of informational containment are best taken up by information theorists; whatever the correct account of these phenomena turns out to be, I will take that account and use it to intepret relevance logic and, by extension, the refined DM theory of extra-mathematical explanation. Importantly, the relation of informational containment is not analysed in terms of explanation. Thus, because the refined DM theory analyses extra-mathematical explanation in terms of informational containment (plus some further features) the threat of circularity is avoided.

Once we interpret relevance using a relation of informational containment, the power of the refined DM theory to solve the irrelevance problem raised in the previous section becomes evident. That problem arises because we can add any and all mathematical claims into a sound argument that seems to be a genuine extra-mathematical explanation and produce a second, sound argument that is then classed as an extra-mathematical explanation as well; the basic DM theory is blind to the distinction between relevant and irrelevant mathematics. We now have a diagnosis of why spurious arguments produced in this manner are spurious. Consider the cicada case. Suppose, as discussed previously, we swap facts about prime numbers out for the fact that $2+2=4.2+2=4$, as we can clearly see, is irrelevant to the fact that we are attempting to explain. What this means is that $2+2=4$ does not contain the right information. That seems exactly right for the case at hand: [P3*] in the specious cicada argument is false precisely because not all the information in the consequent (about the prime numbered life-cycles) is contained in the antecedent of the conditional, since it makes no mention of prime numbers at all.

The following variation on the specious cicada argument can also be handled. Consider $[\mathrm{P} 3 * *]$ :

[P3**] If having a life-cycle period which minimises intersection with other (nearby / lower) periods is evolutionarily advantageous, and prime periods minimise intersection (compared to non-prime periods) and pythagoras' theorem is true then organisms with periodic life-cycles are likely to evolve periods that are prime.

$[\mathrm{P} 3 * *]$, like [P3*], can be used to formulate a specious version of the cicada argument (this is the type of case alluded to in Section Five in which the monotonicity of classical consequence is exploited to produce a junk argument). According to the refined DM theory [P3**] is false 
because not only the information in the consequent is contained in the antecedent of the conditional, since Pythagoras' theorem appears in the antecedent and the consequent makes no mention of geometry at all.

In general, then, the refined DM theory uses the difference between relevant and irrelevant mathematical information as a basis for differentiating between relevant and irrelevant mathematics in any given explanation. The notion of informational containment can also be used to provide a full solution to the genuineness problem raised in Section Three. As we saw, using the REDC to manage the genuineness problem threatens to collapse the distinction between the explanatory and descriptive indispensability of mathematics. But now consider, again, an argument $\alpha$ in which a mathematical claim $M$ is used to derive a non-mathematical claim $P$. Suppose that $M$ in $\alpha$ contains information $I$ that is also contained within the explanandum $P$ and it is in virtue of carrying this information that $P$ is derivable from $M$. Finally, suppose that some part of the information $I$ is descriptive information about a physical system. Call this descriptive information: $i \supseteq I$ (see Section Six for an account of this descriptive information and how it differs from non-descriptive information).

Now, perform the following 'informational test'. Imagine removing $i$ from the total informational content of $M$ whilst holding the rest of the informational content of $M$ fixed. Would $M$ still contain some of the information that is contained within the explanandum $P$ ? If the information in $i$ exhausts the information that $M$ carries regarding $P$, then $M$ fails the informational test and is only contributing descriptive information to the explanation at hand. In this situation, $M$ is playing a merely descriptive role in $\alpha$. If, however, the information in $i$ does not exhaust $I$, then $M$ passes the informational test and carries non-descriptive information regarding the explanandum, information that is used to derive the explanandum and thus explain it. In this situation $M$ plays a non-descriptive role in the explanation.

Using the informational test, conditions for when a DM argument is a genuine extra-mathematical explanation can be given as follows:

[Genuineness] A DM argument is a genuine extra-mathematical explanation when it satisfies the REDC and at least one mathematical claim in that argument passes the informational test.

The genuineness condition enables the differentiation of explanatory and descriptive indispensability. To see this, consider two cases.

First, consider again $\alpha$. Suppose that there is no alternative way of deriving $P$ and that $\alpha$ satisfies the conditions laid down by the basic DM theory as well as the REDC. Given that there are no alternative derivations of $P, M$ is indispensable to explaining $P$. But now suppose that $M$ fails the informational test. Then, despite the fact that $M$ is indispensable to explaining $P$, it is only contributing descriptive information. Suppose, however, that $M$ passes the informational test. Then, $M$ is both indispensable for explaining $P$ and is playing a genuine explanatory role in the explanation.

Second, suppose that $\alpha$ is not the only way of deriving $P$ and, moreover, that there is an alternative way of deriving $P$ from non-mathematical facts alone, it is just that the mathematical derivation is superior to the non-mathematical derivation. Suppose also that $\alpha$ satisfies the conditions laid down by the basic DM theory as well as the REDC. If $M$ fails the informational test, then $M$ is both indispensable and merely descriptive; if $M$ passes the informational test then $M$ is indispensable and genuinely explanatory. As with the previous case, a robust distinction between explanatory and descriptive indispensability is upheld. 


\section{Objections and Replies}

That concludes my presentation of the DM theory. I will now consider and rebut three objections against the view.

\subsection{Against Relevance Logic}

Here's the first objection: any deviation from classical logic should be treated with suspicion. ${ }^{28}$ Such suspicion thus falls upon the refined DM theory as well. There are two things to say here. The first is flat-footed: while classical logic has its place, maybe it just isn't the kind of thing that we should be using to model explanation. This doesn't mean that classical logic needs to be abandoned entirely: classical logic can still legitimately be viewed as the correct account of the consequence relation. Rather, the point is just that classical logic does not provide an appropriate framework for modeling the explanatory relation; relevance logic is needed instead. The second response is more concessive: the refined DM theory can be reformulated entirely within classical logic, should one wish to do so. Here's how: take the space of arguments that are classically valid. Now take the sound arguments from that space that satisfy the tenets of the basic DM theory. Now add to the basic DM theory the following condition:

[Containment] The premises of an extra-mathematical explanation must contain all of the information contained within the conclusion and each premise must contribute some part of that information.

Under this condition, the basic DM theory will classify as explanations all and only those sound arguments that are classified as explanations by the refined DM theory. In essence, what the containment condition does is take the relevance constraint and impose it as a condition on which arguments are explanations, as opposed to a condition on which arguments are valid. The result, however, is the same. So feel free to kick away the ladder.

\subsection{Too Epistemic}

Here's the second objection. According to the refined DM theory, an extra-mathematical explanation is a sound argument in which all of the information contained within the conclusion of that argument is contained within the premises. At core, then, the theory makes use of informational relationships in order to understand explanation. But information, one might think, is an epistemic phenomenon, and so informational containment has too much of an epistemic flavour to form the basis of a theory of extra-mathematical explanation.

What exactly is the objection here? The thought, I suppose, is that information is an epistemic concept, in this sense: information is always information for some knower. Without knowers and thus without conscious beings, there is no information in the world, and thus no explanation. To see why this is a problem it is useful to draw a distinction between two very broad ways of thinking about explanation. Crudely, explanations might be 'in the world' or 'in the head'. An explanation is 'in the world' when it would still exist were there no conscious beings around to attend to it. An explanation is 'in the head', by contrast, when it would not exist were there no conscious beings around to attend to it.

The objection being raised against the refined DM theory, then, is that it treats explanation as something that is 'in the head'. But we all know that explanations are 'in the world'. At the

\footnotetext{
${ }^{28}$ See Williamson ([2013]) for this kind of sentiment.
} 
very least, many of the philosophers to whom this paper is speaking - mathematical realists who motivate their realism by appealing to extra-mathematical explanation - clearly take explanation to be a worldly phenomenon, and for good reason. If a path to mathematical realism is to be forged via extra-mathematical explanation, those explanations had better not be somehow dependent on conscious beings for their existence, on pain of such mind-dependence infecting the mathematical realism at issue.

There are two responses to this objection. The first is just to take issue with the idea that it matters at all whether an explanation is 'in the head' or 'in the world', at least for present purposes. While it is true that I partially motivated the discussion of the DM theory of extra-mathematical explanation via the literature on mathematical realism, I did not claim to be providing a theory of extra-mathematical explanation that may be used by mathematical realists to defend their view. My goal is the more modest one of showing how to understand such explanations, on the assumption that there are some. That goal is perfectly compatible with the resulting theory of extra-mathematical explanation being imbued with an epistemic flavour, and thus being of limited use to realists.

The second, and potentially more satisfying, response is to deny that information is an epistemic concept, at least as it is being used in this paper. Consider the proposition that it is sunny today, in Philadelphia. This proposition seems to carry certain information: it carries information about the weather, on a particular day in a particular city. Moreover, that the proposition carries this information appears to be a completely mind-independent phenomenon. Were there no conscious beings around to attend to the proposition by stating it, asserting it, knowing it, believing it, and so on, it would still be the case that the proposition carries the information in question. More generally we may say that there is a bijective mapping from propositions to information states. I see no reason at all to suppose that this mapping is an epistemic phenomenon, in the sense described above. There just is a fact of the matter about the information that is carried by each proposition. And that is all that the refined DM theory of extra-mathematical explanation ultimately requires. So long as there is a fact of the matter about the information carried by each proposition that appears in a DM argument, the explanation that corresponds to the argument in question will be completely independent of conscious beings in the required sense. Of course, one might think that arguments are some kind of epistemic phenomenon and so not fit to be explanations. If so, then that is a problem for all DN-style theories of scientific explanation (in so far as it is a problem at all) and one that I can reasonably set aside, at least for the time being.

\subsection{Informational Containment}

The third and final objection against the refined DM theory focuses on the notion of 'informational containment' used above. The refined DM theory holds that all of the information contained within the conclusion of an explanation is contained within the premises and, moreover, that each premise must contain part of the information contained within the conclusion. Suppose, then, that we have some claim about the physical world, $P$, that we are attempting to explain. Suppose that $P$ is entailed by physical claim $Q$ plus mathematical claim $M$ such that the resulting sound argument satisfies the tenets of the refined DM theory. It follows that the mathematical claim $M$ contains part of the information contained within $P$. From this it follows that mathematical claims contain information about the physical universe. But, one might argue, that's absurd. Surely mathematical claims contain only information about mathematics; no mathematical claim ever carries physical information!

There are two immediate responses to this objection available. The first response is to just 
deny that mathematical claims carry only mathematical information. But then the question arises: how do we make sense of the idea that mathematical claims contain physical information? I believe we can make sense of this notion by analogy with the laws of nature. Laws of nature are generalisations that provide high-level structural information about physical systems. By 'high-level' information I mean: information about what a system can and cannot do. So, for instance, consider the following rough statement of the second law of thermodynamics: the total entropy of an isolated system always increases over time. This law dictates what can and cannot happen within an isolated system: it tells us that the total entropy of an isolated system cannot increase over time; it tells us that the total entropy can decrease and so on. Call information about what a system can or cannot do: a structural constraint. Laws of nature often encode information about structural constraints.

Importantly, laws of nature are usually highly idealized, in the following sense: they apply primarily to ideal systems (see Cartwright [1983]). For instance, consider again the second law of thermodynamics. Most - if not all — systems in the universe fail to be isolated systems. So the second law appears to be talking about some ideal system (if you don't like the second law of thermodynamics, try Boyle's ideal gas law). An ideal system, however, is plausibly an abstract object. The law, then, tells us what is possible or impossible for this abstract object, at least in the first instance. The law only manages to provide information about a particular physical system derivatively, via some structural mapping relation. Some real, physical systems are structurally very similar to the ideal system to which the law applies. It is in virtue of this structural similarity that the constraints of the ideal system 'flow on' into the real system. That, however, is enough for explanation: because the real system is held under similar structural constraints as the ideal system, things that are not possible in the ideal system are not possible in the real system. Of course, the match is not perfect: what we typically find is that the constraints apply to the real system to some approximation, in virtue of any structural dissimilarities between the real and ideal system. Sometimes, though, the match is perfect and we get very precise answers indeed. ${ }^{29}$

Now, it is not my claim that all laws of nature operate in the manner described above. But clearly some do. Some laws carry information about the physical universe by (i) carrying information about an ideal structure first and (ii) by providing information about a physical system second via a structural mapping between the ideal and physical structures.

Mathematical claims carry information about physical reality in much the same manner. Some mathematical claims - such as those that are used in extra-mathematical explanations - also encode high-level structural information. Consider, for instance, the Bridges of Königsberg. In Königsberg there were once seven bridges connecting a series of islands. It is impossible to cross all seven bridges of Königsberg, passing over every bridge exactly once. Why? The explanation lies in graph theory. If we treat each of the seven bridges as an edge, and each of the islands as a vertex, the seven bridges can be treated as a connected graph. The resulting graph is non-Eulerian, which means that it provably lacks an Eulerian path. An Eulerian path is just a continuous path through a graph that passes over each edge exactly once. The lack of Eulerian paths explains why no-one can cross the seven bridges in the manner described. In this case, mathematical claims about non-Eulerian graphs encode structural information about the kinds of paths that can or cannot obtain through certain mathematical structures. In the first instance, then, these mathematical claims don't yet provide information about any physical system. However, once various structural mappings

${ }^{29}$ For more on the different kinds of structural mapping relations that are available see Bueno and Colyvan ([2011], pp. 348-350). 
are implemented between the mathematical structure and the physical system then mathematical claims come to provide information about the physical system in question, albeit indirectly. The picture, though, is the same as in the nomic case. Mathematical claims, like some laws, provide information about some abstract object in the first instance, and then they provide information about a physical object via a structural mapping between the abstract and physical objects. Note that since structural mappings are, themselves, mathematical objects, at least some mathematical objects carry information about the physical world directly. But this is nothing new, as it seems to be implied in the nomic case anyway.

The preceding discussion leans heavily on Pincock's ([2004, 2007]) structural mapping account of applied mathematics. As Bueno and Colyvan argue, however, Pincock's account leaves little room for mathematics to play an explanatory role in science (Bueno and Colyvan [2011], p. 351). Structural mapping is a form of representation and, as such, a form of description. Accordingly, if the only information that mathematics carries is descriptive information regarding physical structures, then my account of informational containment runs the risk of conflating the descriptive and explanatory roles of mathematics.

The problem may be avoided by appealing to Bueno and Colyvan's inferential conception of applied mathematics. According to the inferential conception, the application of mathematics proceeds in three stages: (i) immersion, whereby a physical system is mapped into a mathematical structure, (ii) inference, whereby inferences are drawn within mathematics about the relevant mathematical structure, and (iii) interpretation, whereby the results of the inferential step are interpreted physically. Note that results are read back down into the physical system via some structural mapping relation between the mathematical and physical structures. Importantly, the structural mapping used in the interpretation step need not be the inversion of the mapping used at the immersion step.

Bueno and Colyvan ([2011], p. 366) maintain that the inferential conception is better suited to handling mathematical explanation than is the structural mapping conception. Although, according to the inferential conception, mathematics is used to describe a physical system via a structural mapping, the mathematics does more than merely describe. Claims about mathematical structures are deduced, and then these claims are used to provide non-descriptive information about physical systems via the interpretation step. Structural mapping is a bridge across which non-descriptive information regarding a physical system may be conveyed.

Bueno and Colyvan's inferential conception therefore provides a useful framework for elucidating the difference between descriptive and non-descriptive physical information (a distinction that was used in my response to the genuineness problem in Section Six). $M$ contains descriptive physical information relevant to $P$ when $M$ contains information about an aspect of some mathematical structure that is mapped into the physical structure corresponding to $P$. $M$ contains non-descriptive physical information relevant to $P$ when $M$ contains information about the physical structure corresponding to $P$ and it does so in virtue of carrying information about an aspect of a mathematical structure and yet that aspect is not a relatum in a mapping relation between the mathematical and physical structures at issue. Importantly, information about what is possible or impossible within a mathematical structure is non-descriptive information; mapping relations relate structures, they don't relate modal facts about structures. Nonetheless, modal facts about one structure can be deduced about another structure in virtue of a mapping. I take this to be one of the central insights of the inferential dimension of Bueno and Colyvan's proposal: there is a difference between the aspects of a mathematical structure that can be mapped to a physical structure, and the aspects of a mathematical structure that cannot be mapped but that nonetheless provide information about a physical structure (in this case, in virtue of a mapping relation). 
In sum, then, mathematical claims, like laws, carry information about physical systems, and they convey this information via structural mappings. The structural mappings do not, however, exhaust the informational content of the relevant mathematical claims. Rather, the structural mappings constitute a point of contact across which non-descriptive information may be conveyed. The information in question being modal information about what a physical system can or cannot do.

Of course, the account is still limited in so far as it won't extend to cases in which mathematics plays an explanatory role in science despite there being no structural mappings in place. As Batterman ([2010]) has urged, however, there are some such cases. For these cases, we might appeal to Batterman's own account of applied mathematics. On this account, mathematics carries information about physical systems indirectly, by carrying information about physical regularities. Mathematics, very roughly, is used to explain why the laws are laws. Mathematics plays this role by carrying information about the limits of regularities:

$[\mathrm{M}]$ any applied mathematical accounts of the robustness of patterns [...] involve the investigation of singularities. To explain and understand the robustness of patterns and regularities, one sometimes needs to focus on places where those very regularities break down. Thus, the existence of patterns or regularities in the world, and our desire to understand and explain them, has led rather straightforwardly to investigate singularities in mathematical limiting operations. (Batterman [2010], p. 21)

A singularity typically arises when a mathematical object is not defined. One way for this to happen - and the case that interests Batterman - is when the limit of a function is both unbounded and the behaviour of the function is different in the infinite limit compared to the finite progression of the function toward the limit. When such singularities are involved, the mathematics of asymptotic operations carries information about a physical regularity. Mathematics carries this information despite the absence of any structural mappings between the mathematics of asymptotic operations and a physical system. It does this because the mathematics limns the boundaries of the physical regularity directly. Batterman's approach is compatible with the broad approach to informational containment sketched above. According to that account, mathematical claims carry information about physical systems in the same way that laws do. Many laws, however, carry information about why it is that other laws hold and on Batterman's view, that is exactly what mathematics does. ${ }^{30}$

As noted, there are two responses to the broad worry about informational containment under discussion. The second response is to deny that mathematical claims ever carry physical information about physical systems. Instead, the reverse is true: claims about physical systems (sometimes) carry mathematical information. Here's the basic idea: it is not just mathematical objects such as numbers, sets, functions, and so on that possess mathematical properties. Rather, some physical objects also possess mathematical properties. This might seem like a strange idea at first, but examples can be given that can help us to better grasp the notion. Here are four such examples. First example: spacetime is thought to be a physical object. But it is also thought that spacetime is continuous. ${ }^{31}$ Continuity, however, is a mathematical property. So spacetimes have mathematical properties. Second example: physical objects possess

${ }^{30}$ As Steiner ([1995], p. 137) notes, this view was defended by Frege.

${ }^{31}$ Spacetime is assumed to be continuous within the general theory of relativity (see Einstein ([2010]). Recent developments in quantum gravity suggest that spacetime may be discrete, however. This is so for (at least) string theory and causal set theory. 
shapes. The property of having a certain shape, however, is a mathematical property, enshrined in geometry and describable algebraically. So physical objects have mathematical properties. Third example: some life-cycles — such as the cycling of the cicada life-cycle length - possess periodicity. Periodicity, however, is a mathematical property, shared by unit cycles more generally, which are abstract objects that may be described within pure mathematics. So some life-cycles have mathematical properties. Fourth example: spacetime has the property of being curved. Curvature, however, is a mathematical property, one that features heavily in the Riemannian geometry that underlies the metric interpretation of general relativity. So spacetimes have mathematical properties.

I don't wish to defend these examples here; they're really just supposed to motivate a much grander thesis about the relationship between the mathematical and the physical. Suppose, however, that the examples are apt and physical systems really do possess mathematical properties. Then it is no surprise that mathematical claims carry information about physical systems, despite not carrying any physical information whatsoever. An extra-mathematical explanation is just a way of bringing the relevant physical and mathematical information together in order to explain some physical-cum-mathematical complex; namely, a complex object consisting of physical and mathematical properties.

The idea that physical systems possess mathematical properties dovetails nicely with Davide Rizza's ([2013]) recent account of applied mathematics. The point of departure for Rizza's account, like Batterman's, is the observation that not all cases of applied mathematics involve structural mappings, and so the structural mapping and inferential conceptions of applied mathematics fail to be fully general. According to Rizza, some cases of applied mathematics operate as follows. First, one identifies some formal property of a physical system. Having identified a formal property of the physical system, one then reasons mathematically about it. These inferences are then used to derive further results regarding the physical system. In other words, on Rizza's view the inferences that are used in the inferential conception of applied mathematics can be conducted in cases where there are no structural mappings. Rather, the target of the inferences are the formal properties that physical systems actually possess. Rizza's account of applied mathematics therefore involves the idea that physical systems sometimes possess mathematical properties, which makes it a useful model for the account of informational containment being proposed.

Now, I recognize that the two responses to the worry about informational containment outlined in this section may inspire more controversy than I have space to fully engage with. For one thing, both responses seem to have a distinctively realist flavour about them: we either need abstract structures or mathematical properties. It pays, then, to offer a third response to the worry. Here it is: providing an account of how it is that mathematical claims contain physical information is everyone's problem; at least, everyone who believes that there are extra-mathematical explanations. In asserting that mathematical claims explain physical claims, it is difficult to see how one can thereby withold commitment from the view that mathematical claims contain information about physical systems. For suppose that mathematical claims contain no such information at all. Then it seems utterly mysterious as to how mathematical claims could ever come to explain physical claims. After all, if mathematical claims contain no information about physical systems then it doesn't seem much of a stretch to say that mathematical claims are incapable of telling us anything about physical systems whatsoever; the two 'realms' appear to be completely distinct. So telling a plausible story about how mathematical claims contain information about physical systems is a rather general concern that any theory of extra-mathematical explanation must ultimately face. Which is just to say that the question of how mathematical claims carry physical information 
is really a general question in applied mathematics, and so will need to be taken up in that context. ${ }^{32}$ I have, however, gestured toward some of the ways in which this might be done, by indicating where my account of informational containment may link up with various theories of mathematical application.

\section{Conclusion}

Here, at last, is a statement of the DM theory, with all the bells and whistles:

\section{The Full-Blown DM Theory of Extra-Mathematical Explanation}

1. Extra-mathematical explanations are sound $\mathbb{R}$-Arguments, where an $\mathbb{R}$-Argument is an argument in which all of the information contained within the conclusion of the argument is contained within the premises, and each premise contributes some part of the information contained within the conclusion.

2. The conclusion of an extra-mathematical explanation is a proposition stating the physical phenomenon to be explained.

3. Among the premises of an extra-mathematical explanation there must be at least one mathematical claim.

4. An extra-mathematical explanation must satisfy the Genuineness Condition.

5. Mathematical claims contain information about physical systems either indirectly, via structural mappings, or directly in virtue of physical objects possessing mathematical properties.

The full-blown DM theory is modeled closely on early DN accounts. The theory will require further refinement to bring it into line with contemporary versions of the DN theory. In fact, achieving such alignment appears quite promising, at least with respect to Jansson's (forthcoming) DN theory. On this theory, the subsumption of an explanandum under a law is understood in terms of inference tickets: inferential licenses that enable one to infer some explanandum from a group of claims that includes a law. According to Restall's (1996) channel theory, the informational containment of $\Delta$ by $\Gamma$ ultimately amounts to the existence of a license to infer the information in $\Delta$ from the information in $\Gamma$, where the inferential licenses themselves are given by the manner in which laws, metaphysical relationships, conventions, and so on control the flow of semantic information. The refined DM theory may therefore be combined with Jansson's version of the DN theory by adopting this channel-theoretic account of the relevance relation, using it as a foundation for explanation.

\section{Acknowledgements}

I am indebted to Alan Baker, Mark Colyvan, Aidan Lyon, Daniel Nolan, Andrew Tedder, and David Ripley for useful discussion of this paper. I am also indebted to the University of Connecticut for hosting me as a visitor during the preparation of this work. Versions of this paper were presented at the Explananza Workshop at the University of Western Australia in

\footnotetext{
${ }^{32}$ See Steiner ([1995]) for an excellent overview of the problems of applied mathematics along with a discussion of the view that mathematical facts are the 'laws of the laws of nature'.
} 
2015 and the Australasian Association of Philosophy Conference in 2015. Research on this article was partly supported by a Humanitites Travelling Fellowship from the Australian Academy of the Humanities (HTF201500015).

\author{
Sam Baron \\ University of Western Australia \\ Philosophy \\ M204 School of Humanities \\ 35 Stirling Highway, Crawley 6009 \\ samuel.baron@uwa.edu.au
}

\title{
References
}

Andersen, R. A. and Belnap, N. [1975]: Entailment: The Logic of Relevance and Necessity, Vol. 1, Princeton: Princeton.

Baker, A. [2005]: 'Are There Genuine Mathematical Explanations of Physical Phenomena?', Mind, 114(454), pp. 223-238.

Baker, A. [2009]: 'Mathematical Explanation in Science', British Journal for the Philosophy of Science, 60(3), pp. 611-633.

Baker, A. [forthcoming]: 'Mathematical Spandrels', Australasian Journal of Philosophy, DOI: http://dx.doi.org/10.1080/00048402.2016.1262881.

Baker, A. and Colyvan, M. [2011]: 'Indexing and Mathematical Explanation', Philosophia Mathematica, 19(3), pp. 323-334.

Bangu, S. I. [2008]: 'Inference to the Best Explanation and Mathematical Realism', Synthese, 160(1), pp. 13-20.

Baron, S. [2014]: 'Optimization and Mathematical Explanation: Doing the Lévy Walk', Synthese, 191(3), pp. 459-479.

Baron, S. [2016a]: 'Explaining Mathematical Explanation', Philosophical Quarterly, 66(264), pp. 458-480.

Baron, S. [2016b]: 'The Explanatory Dispensability of Idealizations', Synthese, 193(2), pp. 365-386.

Baron, S. and Colyvan, M. [2016]: 'Time Enough for Explanation', Journal of Philosophy, 113(2), pp. 61-88.

Barwise, J. and Seligman, J. [1997]: Information Flow: the Logic of Distributed Systems, Cambridge: Cambridge University Press.

Batterman, R. W. [2010]: 'On the Explanatory Role of Mathematics in Empirical Science', British Journal for the Philosophy of Science, 61, pp. 1-25.

Beall, J., Brady, R., Dunn, J. M., Hazen, A. P., Mares, E., Meyer, R. K., Priest, G., Restall, G., Ripley, D., Slaney, J. and Sylvan, R. [2012]: 'On the Ternary Relation and Conditionality', Journal of Philosophical Logic, 41(3), pp. 595-612. 
Bueno, O. and Colyvan, M. [2011]: 'An Inferential Conception of the Application of Mathematics', Noûs, 45(2), pp. 345-374.

Cartwright, N. [1983]: How the Laws of Physics Lie, Oxford: Oxford University Press.

Cohen, J. and Callender, C. [2009]: 'A Better Best System Account of Lawhood', Philosophical Studies, 145, pp. 1-34.

Colyvan, M. [2001]: The Indispensability of Mathematics, Oxford: Oxford University Press.

Colyvan, M. [2002]: 'Mathematics and Aesthetic Considerations in Science', Mind, 111(441), pp. 69-74.

Colyvan, M. [2010]: 'There is No Easy Road to Nominalism', Mind, 119(474), pp. 285-306.

Colyvan, M. [2012]: 'Road Work Ahead: Heavy Machinery on the Easy Road', Mind, 121(484), pp. 1031-1046.

Colyvan, M., Cusbert, J. and McQueen, K. [2017]: 'Two Flavours of Mathematical Explanation', in A. Reutlinger and J. Saatsi (eds), Explanation Beyond Causation, Oxford University Press.

Dretske, F. [1981]: Knowledge and the Flow of Information, Cambridge, Massachusetts: MIT Press.

Einstein, A. [2010]: Relativity: The Special and General Theory, New York: Bibliobazaar.

Forster, M. and Sober, E. [1994]: 'How to Tell when Simpler, More Unified, or Less Ad Hoc Theories will Provide More Accurate Predictions', British Journal for the Philosophy of Science, 45, pp. 1-35.

Goldblatt, R. and Mares, E. D. [2006]: 'An Alternative Semantics for Quantified Relevant Logic', The Journal of Symbolic Logic.

Hempel, C. [1965]: Aspects Scientific Explanation and Other Essays in the Philosophy of Science, New York: Free Press.

Hempel, C. G. and Oppenheim, P. [1948]: 'Studies in the Logic of Explanation', Philosophy of Science, 15(2), pp. 135-175.

Huemer, M. [2009]: 'When is Parsimony a Virtue?', The Philosophical Quarterly, 59(235), pp. 216-236.

Israel, D. and Perry, J. [1990]: 'What is Information?', in P. Hanson (ed.), Information, Language and Cognition, University of British Columbia Press, pp. 1-19.

Jansson, L. [forthcoming]: 'Explanatory Asymmetries: Laws of Nature Rehabilitated', Journal of Philosophy.

Jansson, L. and Tallant, J. [2016]: 'Quantitative Parsimony: Probably for the Better', British Journal for the Philosophy of Science, DOI: https://doi.org/10.1093/bjps/axv064.

Kitcher, P. [1989]: 'Explanatory Unification and the Causal Structure of the World', in P. Kitcher and W. Salmon (eds), Scientific Explanation, University of Minnesota Press, pp. 410-505. 
Lange, M. [2013]: 'What Makes a Scientific Explanation Distinctively Mathematical?', British Journal for the Philosophy of Science, 64(3), pp. 485-511.

Lange, M. [2014]: 'Aspects of Mathematical Explanation: Symmetry, Unity, and Salience', Philosophical Review, 123(4), pp. 485-531.

Lange, M. [forthcoming]: 'Depth and Explanation in Mathematics', Philosophia Mathematica.

Leng, M. [2010]: Mathematics and Reality, Oxford: Oxford University Press.

Lewis, D. [1983]: 'New Work for a Theory of Universals', Australasian Journal of Philosophy, 61(4), pp. 343-377.

Lewis, D. [1986]: 'Causal Explanation', in Philosophical Papers II, Oxford University Press, pp. 214-240.

Lewis, D. [1994]: 'Humean Supervenience Debugged', Mind, 103, pp. 473-490.

Lyon, A. [2012]: 'Mathematical Explanations Of Empirical Facts, And Mathematical Realism', Australasian Journal of Philosophy, 90(3), pp. 559-578.

Lyon, A. and Colyvan, M. [2008]: 'The Explanatory Power of Phase Spaces', Philosophia Mathematica, 16(2), pp. 227-243.

Mancosu, P. [2001]: 'Mathematical Explanation: Problems and Prospects', Topoi, 20(1), pp. 97-117.

Mares, E. D. [2004]: Relevant Logic: A Philosophical Interpretation, Cambridge: Cambridge University Press.

Mares, E. D. [2009]: 'General Information in Relevant Logic', Synthese, 167, pp. 343-362.

Melia, J. [2002]: 'Response to Colyvan', Mind, 111, pp. 75-79.

Peressini, A. F. [1997]: 'Troubles With Indispensability: Applying Pure Mathematics in Physical Theory', Philosophia Mathematica, 5(3), pp. 210-227.

Peressini, A. F. [2010]: 'Numerical Analysis and Its (Invisible?) Role in Mathematical Application', in B. V. Kerkhove, J. D. Vuyst and J. P. V. Bendegem (eds), Philosophical Perspectives on Mathematical Practice, London: College Publications, pp. 331-349.

Pincock, C. [2004]: 'A New Perspective on the Problem of Applying Mathematics', Philosophia Mathematica, 3(12), pp. 135-161.

Pincock, C. [2007]: 'A Role for Mathematics in the Physical Sciences', Noûs, 41, pp. 253-275.

Pincock, C. [forthcoming]: 'Abstract Explanations in Science', British Journal for the Philosophy of Science.

Priest, G. [2002]: Beyond the Limits of Thought, Oxford: Oxford University Press.

Railton, P. [1978]: 'A Deductive-Nomological Model of Probabilistic Explanation', Philosophy of Science, 45, pp. 206-226. 
Railton, P. [1981]: 'Probability, Explanation, and Information', Synthese, 48, pp. 233-256.

Restall, G. [1996]: 'Information Flow and Relevant Logics', in J. S. . D. W. hl (ed.), Logic, Language and Computation, CSLI, pp. 463-477.

Restall, G. and Dunn, J. M. [2002]: 'Relevance Logic', in D. Gabbay and F. Guenther (eds), The Handbook of Philosophical Logic, Kluwer, pp. 1-136.

Rice, C. [2015]: 'Moving Beyond Causes: Optimality Models and Scientific Explanation', Noûs, 49(3), pp. 589-615.

Rizza, D. [2013]: 'The Applicability of Mathematics: Beyond Mapping Accounts', Philosophy of Science, 80, pp. 398-412.

Routley, R. and Meyer, R. K. [1972a]: 'Semantics of Entailment-II', Journal of Philosophical Logic, 1, pp. 53-73.

Routley, R. and Meyer, R. K. [1972b]: 'Semantics of Entailment-III', Journal of Philosophical Logic, 1, pp. 192-208.

Routley, R. and Meyer, R. K. [1973]: 'Semantics of Entailment', in H. Leblanc (ed.), Truth, Syntax and Modality, Proceedings of the Temple University Conference on Alternative Semantics, pp. 194-243.

Saatsi, J. [2011]: 'The enhanced indispensability argument: representational vs. explanatory role of mathematics in science', British Journal for the Philosophy of Science, 62, pp. $143-154$.

Saatsi, J. [2012]: 'Mathematics and Program Explanations', Australasian Journal of Philosophy, 90, pp. 579-584.

Saatsi, J. [forthcoming]: 'On the "Indispensable Explanatory Role” of Mathematics', Mind.

Salmon, W. [1984]: Scientific Explanation and the Causal Structure of the World, Princeton: Princeton University Press.

Schrenk, M. [2014]: 'Better Best Systems and the Issue of CP-Laws', Erkenntnis, 79, pp. 1787-1799.

Skow, B. [forthcoming]: 'Are There Non-Causal Explanations (of Particular Events)?', British Journal for the Philosophy of Science.

Skyrms, B. [1992]: 'Discussion: Hypothetico-Deductivism is Hopeless by Clark Glymour;Relevance Logic Brings Hope to Hypothetico-Deductivism by C. Kenneth Waters;Discussion: Truth, Content, and the Hypothetico-Deductive Method by Thomas R. Grimes', The Journal of Symbolic Logic, 57(2), pp. 756-758.

Sober, E. [2016]: Ockham's Razors, Cambridge: Cambridge University Press.

Steiner, M. [1992]: 'Mathematical Rigor in Physics', in M. Detlefsen (ed.), Proof and Knowledge in Mathematics, London: Routledge, pp. 158-170.

Steiner, M. [1995]: 'The Applicabilities of Mathematics', Philosophia Mathematica, 3(3), pp. 129-156. 
Strevens, M. [2008]: Depth: An Account of Scientific Explanation, Cambridge, Massachusetts: Harvard University Press.

Waters, K. [1987]: 'Relevance Logic Brings Hope to Hypothetico-Deductivism', Philosophy of Science, 54(3), pp. 453-464.

Williamson, T. [2013]: Modal Logic as Metaphysics, Oxford University Press.

Woodward, J. [2003]: Making Things Happen: A Theory of Causal Explanation, Oxford: Oxford University Press.

Woodward, J. and Hitchock, C. [2003]: 'Explanatory Generalizations, Part 1: A Counterfactual Account', Nô̂s, 37(1), pp. 1-24. 